\title{
Successful control of a hospital-wide outbreak of OXA- 48 producing Enterobacteriaceae in the Netherlands, 2009 to 2011
}

M J Dautzenberg (m.j.d.dautzenberg@umcutrecht.nl)1,2,3, J M Ossewaarde ${ }^{1}, M$ E de Kraker ${ }^{4}$, A van der Zee ${ }^{1}, \mathbf{S}$ van Burgh ${ }^{1}$,

S C de Greeff4, H A Bijlmer ${ }^{4}$, H Grundmann ${ }^{4,5}$, J W Cohen Stuart ${ }^{2}$, A C Fluit ${ }^{2}$, A Troelstra ${ }^{2}$, M J Bonten ${ }^{2,3}$

1. Department of Medical Microbiology, Maasstad Ziekenhuis, Rotterdam, the Netherlands

2. Department of Medical Microbiology, University Medical Center Utrecht, Utrecht, the Netherlands

3. Julius Center for Health Sciences and Primary Care, University Medical Center Utrecht, Utrecht, the Netherlands

4. Center for Infectious Disease Control, National Institute for Public Health and the Environment (RIVM), Bilthoven, the Netherlands

5. University Medical Center Groningen, University of Groningen, the Netherlands

Citation style for this article:

Dautzenberg MJ, Ossewaarde JM, de Kraker ME, van der Zee A, van Burgh S, de Greeff SC, Bijlmer HA, Grundmann H, Cohen Stuart JW, Fluit AC, Troelstra A, Bonten MJ. Successful control of a hospital-wide outbreak of OXA-48 producing Enterobacteriaceae in the Netherlands, 2009 to 2011. Euro Surveill. 2014;19(9):pii=20723. Available online: http://www.eurosurveillance.org/ViewArticle.aspx?Articleld=20723

Article submitted on 19 September 2013 / published on 6 March 2014

On 31 May 2011, after notification of Klebsiella pneumoniae $(\mathrm{KP})_{\mathrm{OXA}-48 ; \mathrm{CTX}-\mathrm{M}-15}$ in two patients, nosocomial transmission was suspected in a Dutch hospital. Hospital-wide infection control measures and an outbreak investigation were initiated. A total of 72,147 patients were categorised into groups based on risk of OXA-48 colonisation or infection, and 7,527 were screened for Enterobacteriaceae ${ }_{\text {OXA-48 }}$ by polymerase chain reaction (PCR). Stored KP isolates $(n=408)$ were retrospectively tested for OXA-48 and CTX-M-1 group extended-spectrum beta-lactamases (ESBL). $285 \mathrm{KP}$ isolates from retrospective and prospective patient screening were genotyped by amplified fragment length polymorphism (AFLP). 41 isolates harbouring different Enterobacteriaceae species were analysed by plasmid multilocus sequence typing (pMLST). No nosocomial transmission of Enterobacteriaceae ${ }_{\text {OXA-48 }}$ was detected after 18 July 2011. Enterobacteriaceae ${ }_{\text {OXA-48 }}$ were found in 118 patients (KP $(n=99)$, Escherichia coli $(n=56), \geq 1$ Enterobacteriaceae ${ }_{0 \times A-48}$ species $\left.(n=52)\right)$, of whom 21 had clinical infections. 39/41 (95\%) of OXA-48 containing plasmids were identical in pMLST. Minimum inhibitory concentrations (MICs) of $\mathrm{KP}_{\text {OXA-48 }}$ and $E$. coli $_{\text {OXA-48 }}$ for imipenem and meropenem ranged from $\leq 1$ to $\geq 16 \mathrm{mg} / \mathrm{L}$, and $153 / 157$ (97\%) had MIC $>0.25 \mathrm{mg} / \mathrm{L}$ for ertapenem. AFLP identified a cluster

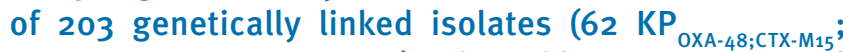
$\left.107 \mathrm{KP}_{\text {CTX-M-15 }} ; 34 \mathrm{KP}_{\text {OXA-48 }}\right)$. The 'oldest' $\mathrm{KP}_{\text {CTX-M-15 }}$ and $\mathrm{KP}_{\text {OXA-48 }}$ Clonal types originated from February 2009 and September 2010, respectively. The last presumed

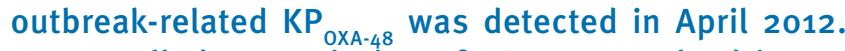
Uncontrolled transmission of $\mathrm{KP}_{\mathrm{CTX}-\mathrm{M}-15}$ evolved into a nosocomial outbreak of $\mathrm{KP}_{\mathrm{OXA}-48 ; \mathrm{CTX}-\mathrm{M}_{15}}$ with large phenotypical heterogeneity. Although the outbreak was successfully controlled, the contribution of individual containment measures and of the hospital relocating into a new building just before outbreak notification was impossible to quantify.

\section{Introduction}

The number of infections caused by Enterobacteriaceae producing extended-spectrum beta-lactamases (ESBL), such as for example those with CTX-M-1 group ESBL and/or carbapenemases is rapidly increasing worldwide [1-3]. Carbapenem resistance in Enterobacteriaceae is mainly caused by production of one of three groups of carbapenemases: Ambler class A (Klebsiella pneumoniae carbapenemase (KPC)), B (metallo-beta-lactamases, e.g. Verona integron-encoded metallo-beta-lactamase (VIM), New Delhi metallo-betalactamase (NDM)), and D (oxacillinases, e.g. OXA-48), usually in combination with ESBL-production.

OXA-48 oxacillinase was identified for the first time in a K. pneumoniae isolate from Istanbul, Turkey in 2001 [4], and patients infected or carrying such bacteria have been reported from Asia [5], north Africa [6-8], South Africa [9], Europe [10-12] and north America [13]. OXA-48 has also been described in Escherichia coli, Enterobacter cloacae, and Citrobacter freundii and hospital outbreaks have been reported across Europe and the northern part of Africa [6,7,10-12,14,15]. Hospital outbreaks in western-European countries, such as France and Spain have been linked to transfer of patients from hospitals in endemic countries, such as Turkey and Morocco $[8,12,16,17]$.

In 2008 sporadic events of $K$. pneumoniae with ESBL phenotypes occurred in a Dutch hospital (hospital A). Subsequently, in February 2009 two clonal type

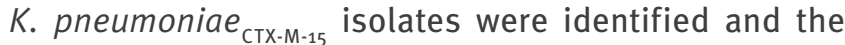
occurrence of this clonal type gradually increased since, despite implementation of several control measures to interrupt transmission.

In May 2011, K. pneumoniae OXA-48;CTX-M-15 was notified in two patients after discharge from hospital A. As 
K. pneumoniae ${ }_{\mathrm{OXA}-48}$ were only detected only sporadically in Dutch hospitals, the coincidence of this finding was considered highly suggestive for nosocomial transmission. This initiated a retrospective and prospective investigation to determine the potential spread and the number of patients colonised and/or infected with Enterobacteriaceae harbouring OXA-48. There was, in parallel, implementation of a hospital-wide outbreak containment strategy. Moreover microbiological studies were undertaken to determine associations between $K$. pneumoniae oxA-48 and previously identified K. pneumoniae ${ }_{\mathrm{CTX}-\mathrm{M}-15}$ isolates. Here, we describe the sequence of events, effects of intervention and results from microbiological investigation of the, to our knowledge, largest nosocomial outbreak of $K$. pneumoniaeOXA-48 in Europe so far.

\section{Methods}

\section{Setting}

Hospital A is a secondary care hospital. Until 16 May 2011 care was provided at two locations. Acute care was given in location 1, with 340 beds and a 12-bed intensive care unit (ICU), and non-acute care was concentrated in location 2 with 242 beds and a seven-bed ICU. On 16 May 2011, the entire hospital was moved to a new building physically separated from the two past locations. Coincidentally to the move, the total number of beds provided by the hospital changed, whereby the new building comprises 602 beds and 16 ICU beds.

Following the notification of two patients on 31 May 2011, with K. pneumoniae ${ }_{\text {OXA-48:CTX-M15 }}$ growing from clinical cultures, after hospitalisation in hospital $A$, an outbreak investigation was initiated on 1 June 2011.

\section{Infection control measures}

As soon as the outbreak investigation started, the hospital received immediate assistance in outbreak control from four members of the National Institute of Health and the Environment and from three experts of the department of Medical Microbiology of the University Medical Center (UMC) Utrecht from 30 June 2011 onwards. The Dutch guideline for highly resistant microorganisms (HRMO) was implemented throughout the hospital starting from 30 June [18]. This included patient screening for Enterobacteriaceae ${ }_{0 \times A-48}$ and preemptively isolating patients from defined risk categories (see below) upon admission. Patients with HRMO were also isolated.

Cohorting of dedicated nursing staff was not applied. However, communication between the departments of medical microbiology and infection prevention and other healthcare personnel was intensified. Adherence to basic infection control measures was pursued through regular instruction meetings at hospital wards, but was not quantified. During the initial phases of outbreak control, between 7 and 27 June 2011, all ICU staff members ( 80 nurses and 16 physicians) were screened using both throat and rectal swabs, and none were found positive for OXA-48. In addition, 26 environmental samples were obtained in ICU in July 2011 (including air filters and objects in rooms of OXA-48 carriers). As these environmental samples tested negative for OXA-48, cleaning procedures, which after 1 June had remained as prior to the outbreak, were not subsequently changed and elimination of colonisation in patients was not attempted. There was no intervention on antibiotic stewardship, and patients with infections were treated according to standard antimicrobial treatment policy. Healthcare personnel and environmental screening was not repeated.

\section{Categorisation of patients}

Based on the emergence of $K$. pneumoniae with ESBL phenotype in hospital A since early 2009, start and end of the outbreak period were - arbitrarily - defined as 1 July 2009 and 18 July 2011, respectively (see results).

Patients with a clinical culture (taken because of a clinical suspicion of infection by treating physician) or screening culture (taken with the purpose of detecting OXA-48 carriage, without a clinical suspicion of infection) taken after 1 January 2009, containing PCRconfirmed OXA-48 positive Enterobacteriaceae, excluding Shewanella spp., were considered OXA-48 carriers. The status of OXA-48 carrier was maintained unless six consecutive screening cultures from the patient performed every two months were negative (i.e. 1 year negative).

Other patients were classified in three groups based on risk of OXA-48 colonisation or infection (Table 1).

- High-risk patients comprised (i) patients who had shared a hospital room with an OXA-48 carrier during the outbreak period or had been admitted to a room from which an OXA-48 carrier had been discharged within the previous two hours and (ii) all patients identified since 1 July 2009 with Enterobacteriaceae having minimum inhibitory concentrations (MICs) for imipenem $\geq 2 \mathrm{mg} / \mathrm{L}$ or meropenem $\geq 0.5 \mathrm{mg} / \mathrm{L}$, as detected by the Vitek 2 system (bioMérieux, Marcy l'Etoile, France). If the later patients had been hospitalised, individuals who had shared a room with such patients or had been in the same room within two hours after discharge and/or transfer to another room of such patients were also considered as belonging to the high risk group.

- Medium-risk patients comprised patients who had been admitted to hospital A during the outbreak period, but did not meet high-risk criteria.

- Low-risk patients included patients who had not been hospitalised in hospital A during the outbreak period. These patients were considered not to be exposed to OXA-48 carriers.

Patients were flagged accordingly in the electronic patient record system, which provided automatic popups for OXA-48 carriers, high-risk, and medium-risk patients, in order to allow adequate precautions as 
described in the sections 'screening' and 'infection control measures'.

\section{Screening}

\section{Retrospective screening}

Retrospective screening included PCR-based screening of OXA-48 and CTX-M group 1 ESBL of all $K$. pneumoniae isolates that had been stored since 1 January 2009. There was protocolised storage of all clinical isolates from blood cultures (irrespective of antibiotic susceptibility) and of all clinical isolates with an ESBL phenotype, supplemented with isolates with otherwise notable antibiograms. Retrospective screening started mid-May 2011 as the presence of carbapenemases was, based on phenotype, suspected in some clinical K. pneumoniae isolates.

\section{Prospective screening}

Prospective screening for Enterobacteriaceae ${ }_{0 \times A-48}$ included obtaining swabs (Amies Agar Gel 108C,
Copan) on three consecutive days from rectum, throat and possible infection sites, such as wounds, sputum, and urine, when applicable. After outbreak notification, hospitalised patients were screened using conventional culture techniques, and high-throughput PCR-based screening for OXA-48 started on 10 June 2011.

- Patients in the high-risk group were screened on readmission when hospitalised, and if not hospitalised through post-discharge screening. For this, nonhospitalised high-risk patients received information and material for sampling that could be returned through mail. High-risk patients were not recalled to the hospital to be screened.

- Patients in the medium-risk group were screened on readmission when hospitalised.

- Patients in the low risk group were not screened on admission.

To detect unnoticed OXA-48 transmission in the hospital, all patients hospitalised for more than seven

\section{TABLE 1}

Risk group classification of patients during an outbreak of OXA-48 positive Enterobacteriaceae in a hospital, Rotterdam, the Netherlands, 2009-2011

\begin{tabular}{|c|c|c|c|c|c|}
\hline $\begin{array}{l}\text { Risk group } \\
\text { (number in } \\
\text { group) }\end{array}$ & $\begin{array}{l}\text { Screened } \\
\quad N(\%)\end{array}$ & $\begin{array}{l}\text { OXA-48 } \\
\text { positive } \\
\mathrm{N}(\% \text { of } \\
\text { screened })\end{array}$ & Definition & Identification strategy & Barrier precautions \\
\hline $\begin{array}{l}\text { OXA-48 carrier } \\
(118)\end{array}$ & NA & NA & $\begin{array}{l}\text { Patients with a clinical culture (taken because } \\
\text { of a clinical suspicion of infection by treating } \\
\text { physician) or screening culture (taken with the } \\
\text { purpose of detecting OXA-48 carriage, without } \\
\text { a clinical suspicion of infection) taken after } 1 \text { Jan } \\
\text { 2009, containing PCR-confirmed OXA-48 positive } \\
\text { Enterobacteriaceae, excluding Shewanella spp. } \\
\text { The status of OXA-48 carrier was maintained } \\
\text { unless six consecutive screening cultures from } \\
\text { the patient performed every two months were } \\
\text { negative (i.e. } 1 \text { year negative) }\end{array}$ & NA & $\begin{array}{l}\text { Single room } \\
\text { Gowns and gloves } \\
\text { Masks during high } \\
\text { risk interventions }\end{array}$ \\
\hline $\begin{array}{l}\text { High-risk } \\
(4,722)\end{array}$ & $3,394(72)$ & $35(1)$ & $\begin{array}{l}\text { Shared room with or was in same room within } 2 \\
\text { hours after discharge and/or transfer to another } \\
\text { room of OXA- } 48 \text { carrier } \\
\text { Had Enterobacteriaceae in clinical sample with } \\
\text { MIC for imipenem } \geq 2 \mathrm{mg} / \mathrm{L} \text { or meropenem } \geq 0.5 \\
\mathrm{mg} / \mathrm{L} \text {, or shared room with or was in same room } \\
\text { within } 2 \text { hours after discharge and/or transfer to } \\
\text { another room of such a patient }\end{array}$ & $\begin{array}{l}\text { Screening on } \\
\text { readmission when } \\
\text { hospitalised } \\
\text { Post-discharge screening } \\
\text { when not hospitalised } \\
\text { From Jun } 2011 \text { to Jan } 2012 \\
\text { weekly screening when } \\
\text { hospitalised }>7 \text { days }\end{array}$ & $\begin{array}{l}\text { Single room }{ }^{\mathrm{a}} \\
\text { Gowns and gloves } \\
\text { Masks during high } \\
\text { risk interventions }\end{array}$ \\
\hline $\begin{array}{l}\text { Medium-risk } \\
(67,361)\end{array}$ & $4,133(6)$ & $8(<1)$ & $\begin{array}{c}\text { Was admitted to hospital A during outbreak period } \\
\text { (1 Jul 2009-18 Jul 2011), and did not fulfil criteria } \\
\text { for OXA-48 carrier or high-risk }\end{array}$ & $\begin{array}{c}\text { Screening on } \\
\text { readmission when } \\
\text { hospitalised } \\
\text { From Jun } 2011 \text { to Jan } 2012 \\
\text { weekly screening when } \\
\text { hospitalised >7 days }\end{array}$ & $\begin{array}{l}\text { Cohorting }^{\text {b }} \\
\text { General } \\
\text { precautions }\end{array}$ \\
\hline Low-risk (ND) & 1,921 (NA) & $4^{c}(<1)$ & $\begin{array}{l}\text { Was not admitted to hospital A during outbreak } \\
\text { period (1 Jul 2009-18 Jul 2011) }\end{array}$ & $\begin{array}{l}\text { Point-prevalence surveys } \\
\text { From Jun } 2011 \text { to Jan } 2012 \\
\text { weekly screening when } \\
\text { hospitalised }>7 \text { days }\end{array}$ & $\begin{array}{l}\text { Cohorting }^{\mathrm{b}} \\
\text { General } \\
\text { precautions }\end{array}$ \\
\hline
\end{tabular}

MIC: minimum inhibitory concentration; NA: not applicable; ND: not determined; PCR: polymerase chain reaction.

a Single room or cohorting with other high-risk patients, if insufficient availability of single rooms.

b Cohorting with patients in the same risk group.

c Not epidemiologically linked to outbreak. 
days, including low risk patients and high/medium risk patients, were screened weekly from June 2011 onwards (until January 2012).

\section{Microbiology}

Screening patients and isolates for OXA- 48

Screening swabs were inoculated - overnight - in broth containing ertapenem $(0.125 \mathrm{mg} / \mathrm{L})$.

A robotised PCR-procedure was designed using the following primers: OXA-48 Forward 5'-GCGTGGTTAAGGATGAACAC-3', OXA-48 Reverse 5'-CATCAAGTTCAACCCAACCG-3', and OXA-48 probe, labelled with 6-carboxyfluorescein (FAM), 5'-FAMAGCCATGCTGACCGAAGCCAATG-3', generating a DNA fragment of $438 \mathrm{bp}$. This PCR was modified from Poirel et al. [19].

In case of PCR positivity, isolates, regardless of the retrospective or prospective sampling procedure, were cultured on carbapenem-resistant Enterobacteriaceae (CRE) agar (Oxoid Brilliance CRE Agar) and McConkey agar (Oxoid), and the presence of OXA-48 was reconfirmed by PCR in every morphologically different isolate, to a maximum of six colonies (Figure 1). Validation of this procedure before large-scale application yielded $100 \%$ specificity.

MICs for amikacin, cefepime, cefotaxime, ciprofloxacin, gentamicin, imipenem, meropenem, and trimethoprim/sulfamethoxazole were determined by automated

\section{FIGURE 1}

Schematic representation of polymerase chain reactionbased screening procedures used during an outbreak of OXA-48 positive Enterobacteriaceae in a hospital, Rotterdam, the Netherlands, 2009-2011

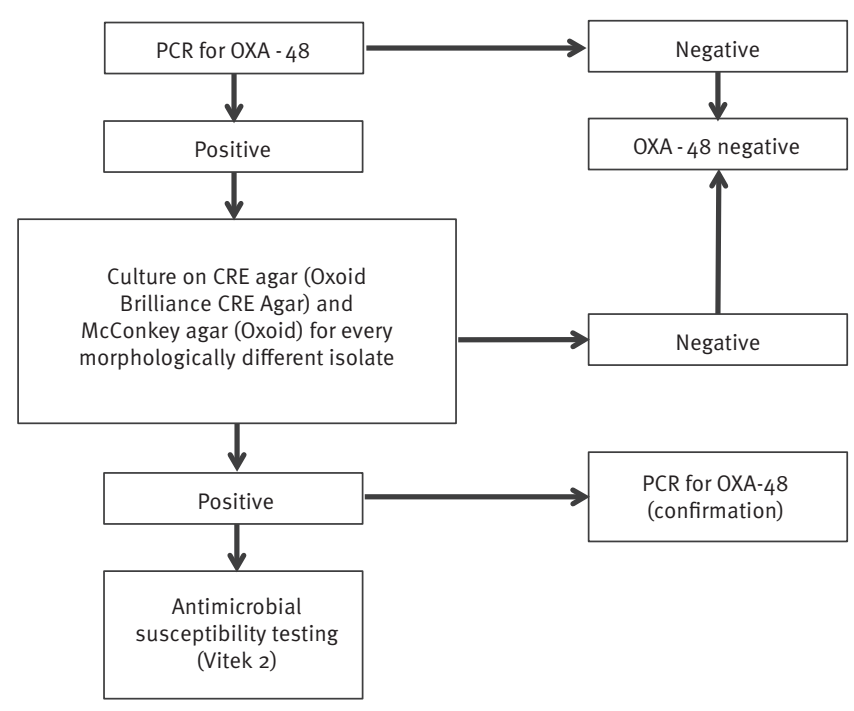

PCR: polymerase chain reaction; CRE: carbapenem-resistant Enterobacteriaceae. susceptibility testing (Vitek 2) and MIC for ertapenem was determined by Etest (bioMérieux, Marcy l'Etoile, France).

Screening OXA-48 positive isolates for extendedspectrum beta-lactamases phenotype

For all isolates found to be OXA-48 positive by the method above (Figure 1), ESBL phenotype was verified. ESBL phenotype was defined as a MIC $\geq 1 \mathrm{mg} / \mathrm{L}$ for cefotaxime, or ceftazidime, and confirmation results of disk diffusion using cefepime, cefotaxime, and ceftazidime, with and without clavulanic acid according to national guidelines [20].

Screening Klebsiella pneumoniae isolates with extendedspectrum beta-lactamases for CTX-M group 1

Regardless of whether they were obtained from retrospective of prospective screening, $K$. pneumoniae isolates with ESBL phenotype (only $1^{\text {st }}$ isolate per patient) were tested by PCR for CTX-M group 1 (primers: Forward 5'-GCTGGACTGCCTGCTTCCT-3', Reverse 5'- CGTTGGTGGTGCCATAG(C/T)CA-3', and minor groove binder (MGB) probe 5'-CCGCTGCCGGTCTTATC-MGB-3'), and $C T X-M$ group 1 isolates were sequenced or tested with gene-specific PCR for CTX-M-15.

Molecular investigation of the outbreak

CTX-M group 1 and/or OXA-48 K. pneumoniae isolates were genotyped using amplified fragment length polymorphism (AFLP) [21,22]. Similarity was defined as $\geq 85 \%$ resemblance (Dice). AFLP typing was performed for all $K$. pneumoniae isolates included in retrospective screening, as well as for $K$. pneumoniae isolates detected through prospective screening.

A plasmid multilocus sequence typing (pMLST) scheme was developed for typing of bla $_{0 \times A-48}$ containing plasmids. Plasmid sequences were filtered from the reported whole genome sequence of the outbreakrelated K. pneumoniae 1191100241 (Project 71587 GenBank Assignment: AFXHoooooooo). Four contigs were identified and PCR and conventional sequencing enabled the determination of the order and orientation of the contigs as well as the closure of the remaining gaps in the sequence. A basic local alignment search tool (BLAST) search identified four plasmids with a similar backbone: $\mathrm{pCTXM} 360 \mathrm{~K}$. pneumoniae (GenBank accession number: EU938349.1); pNDMHK E. coli (GenBank accession number: HQ451074.1); pEL6o Erwinia amylovora (GenBank accession number: AY422214.1); pCTX-M3 C. freundii (GenBank accession number: AF550415.2). Initial analysis of these plasmids showed 11 potential sequences with sequence variation.

The four sequences with most sequence variation were selected and their usefulness for typing was validated in 13 OXA-48 encoding plasmids: Plasmids from six isolates were from the current outbreak (as was the whole genome sequenced isolate), one isolate was possibly outbreak related, but from a different hospital, and six 
isolates were obtained from six different geographic locations in the Netherlands, and considered unrelated to this outbreak. Four of the six plasmids from the isolates unrelated to the outbreak had clear sequence differences compared to the K. pneumoniae 1191100241 OXA-48 plasmid, whereas only one of the six plasmids obtained from outbreak-related isolates had clear differences. The plasmid from the isolate that was possibly outbreak related but from a different hospital had a nucleotide difference. From these data we concluded that the chosen sequences provided sufficient resolution.

We did not determine the incompatibility group or performed transfer experiments because the incompatibility class could be deduced from the whole genome sequence data and pMLST is more discriminatory than the determination of the incompatibility group. Isolates for plasmid typing were selected from 15 patients who had at least two different OXA-48 containing Enterobacteriaceae, of which one $K$. pneumoniae, maximising diversity in species.

\section{Results}

\section{Infection control measures}

Prior to the notification of the outbreak of OXA-48 positive Enterobacteriaceae, a certain number of steps had already been taken to contain $K$. pneumoniae with ESBL phenotype, which had been detected in the hospital from 2009 onwards (Table 2). After notification in May 2011 of $K$. pneumoniaeCTX-M-15 simultaneously positive for OXA-48, in clinical cultures of two patients who had been recently discharged from hospital $A$, an outbreak team was assembled on 1 June. PCR procedures for OXA-48 screening were fully operational on 10 June 2011. On 18 July 2011, all infection control measures as described in the 'Infection control' section had been implemented and accurate flagging of all patients in hospital database systems had been realised.

\section{Screening}

Retrospective screening

The identification of two patients with $K$. pneumoniaeCTX-M-15;OXA-48 by other laboratories in March and April 2011 alerted to the outbreak upon notification on 31 May 2011. These two OXA-48 carriers are included in the group of the retrospectively screened patients in further analyses.

Furthermore, retrospective evaluation of all stored K. pneumoniae isolates $(\mathrm{n}=408)$ revealed 85 isolates harbouring OXA-48, from 43 patients (Figure 2); 77 isolates were also positive for CTX-M-15. The 'oldest' OXA48 isolate (also harbouring CTX-M-15) was identified in a clinical sample obtained on 10 September 2010.

Detection of OXA-48 carriers by prospective hospital screening or clinical sample testing

Accurate flagging of all patients in the hospital database systems as 'low', 'medium' or 'high' risk was realised on 18 July 2011. Between outbreak detection (31 May 2011) and full implementation of the flagging system (18 July 2011) prospective hospital screening or testing of clinical samples revealed OXA-48 carriage in 30 hospitalised patients (Figure 2). The 30 patients detected through prospective screening or clinical samples were different from the 45 patients found by retrospective screening. Two of these 30 patients were

\section{TABLE 2}

Control measures for extended-spectrum beta-lactamases (ESBL) and OXA-48 producing Enterobacteriaceae during an outbreak of OXA-48 positive Enterobacteriaceae, Rotterdam, the Netherlands, 2009-2011

\begin{tabular}{|c|c|}
\hline Date & Measure \\
\hline Jun 2010 & $\begin{array}{l}\text { - Separation of two ICU-units } \\
\text { - Increased attention for hygiene measures } \\
\text { - Enhanced cleaning of ICU-units }\end{array}$ \\
\hline Aug 2010 & Start twice weekly throat and rectum screening for $K$. pneumoniae with ESBL phenotype in ICU patients \\
\hline Sep 2010 & Start making of daily summary of all relevant microorganisms on ICU \\
\hline Oct 2010 & Hand hygiene promotion on ICU \\
\hline Mar 2011 & 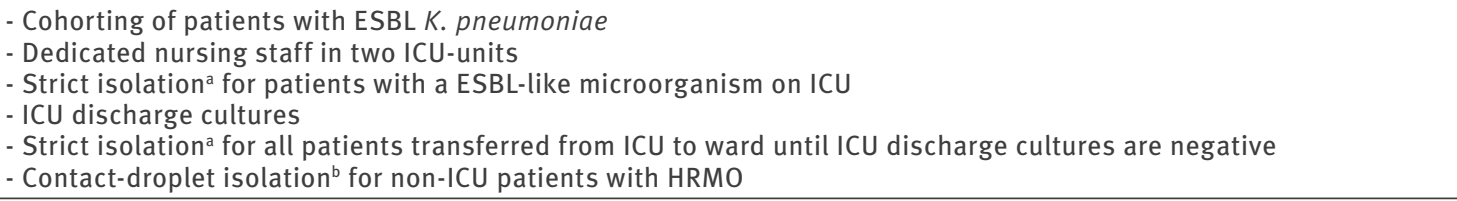 \\
\hline May 2011 & Start of SDD in ICU prior to the relocation of the hospital into a new building \\
\hline Jun 2011 & $\begin{array}{l}\text { Notification of OXA-48 positive Enterobacteriaceae outbreak. Start PCR based screening for OXA-48 and pre-emptive } \\
\text { isolation as described in Table } 1\end{array}$ \\
\hline July 2011 & 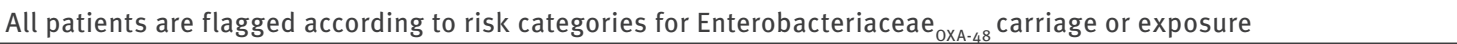 \\
\hline
\end{tabular}

ESBL: extended spectrum beta-lactamase; HRMO: highly resistant microorganism; ICU: intensive care unit; PCR: polymerase chain reaction; SDD: selective decontamination of the digestive tract.

a Strict isolation: single isolation room, use of gown, gloves and mask.

b Contact-droplet isolation: single room, use of gloves and mask. 
identified in August, but both had been hospitalised from before 18 July.

In total, 72,147 patients had been admitted during the outbreak period (1 July 2009 until 18 July 2011). By the time flagging of patients was completely operational (18 July 2011), 64 of these patients had already been categorised as 0XA-48 carriers. Of the 72,083 remaining, 4,722 were classified as high-risk and 67,361 as medium-risk, of whom 3,394 (72\%) and 4,133 (6\%) were screened respectively post-discharge or on readmission (Table 1). This yielded 43 newly identified OXA48 carriers ( 35 from the high-risk and eight from the medium-risk patient group). The last patient with OXA48 was detected through post-discharge screening on 14 April 2012.

In all, of 73 OXA-48 carriers detected prospectively, five were detected via clinical cultures while 68 were identified through screening. All 68 had documented rectal carriage and 13 (19\%) also had throat carriage.

In total, prospective and retrospective analysis led to 118 OXA-48 carriers being found (Figure 2 ).

In a period after the outbreak starting from 18 July 2011 until 18 July 2012, 1,921 patients (not admitted between 1 July 2009 up to 17 July 2011 included) were screened for OXA-48 carriage after seven days hospitalisation or during point-prevalence surveys. In this period three patients with OXA-48 Enterobacteriaceae (one $C$. freun$d i$, one $E$. coli and one $K$. pneumoniae) were detected through point-prevalence survey. Another patient had OXA-48 K. pneumoniae in a clinical culture obtained at hospital admission. All findings could not be epidemiologically linked to the outbreak and both $K$. pneumoniae isolates did not belong to the outbreak strain based on AFLP-typing. These episodes were, therefore, considered new introductions.

\section{Patient characteristics}

In 21 of 118 patients (18\%) Enterobactericeae ${ }_{\text {OXA-48 }}$ was associated with clinical signs of infection and all-cause 30-day mortality was $29 \%(n=6)$ and $13 \%(n=13)$ for patients with infection $(n=21)$ and colonisation only $(n=97)$, respectively (Table 3$)$.

Before the transmigration of both hospital locations (16 May 2011), 107 patients with OXA-48 Enterobacteriaceae had been hospitalised and they had a median of seven roommates per admission (interquartile range (IQR): 3-15), which generated a median of 1.04 (IQR: 0.6-3.0) new contact patients per admission day. After transmigration (and before 18 July 2011) 49 OXA-48 carriers were hospitalised with a median of o roommates (IQR: $0-4)$, generating a median of o (IQR: 0-1.56) new contacts per admission day ( $p<0.001)$.

\section{FIGURE 2}

Epidemic curve of detection date of first OXA-48 positive isolate per patient during an outbreak of OXA-48 positive Enterobacteriaceae, Rotterdam, the Netherlands, 2009-2011 ( $\mathrm{n}=118$ patients) ${ }^{\mathrm{a}}$

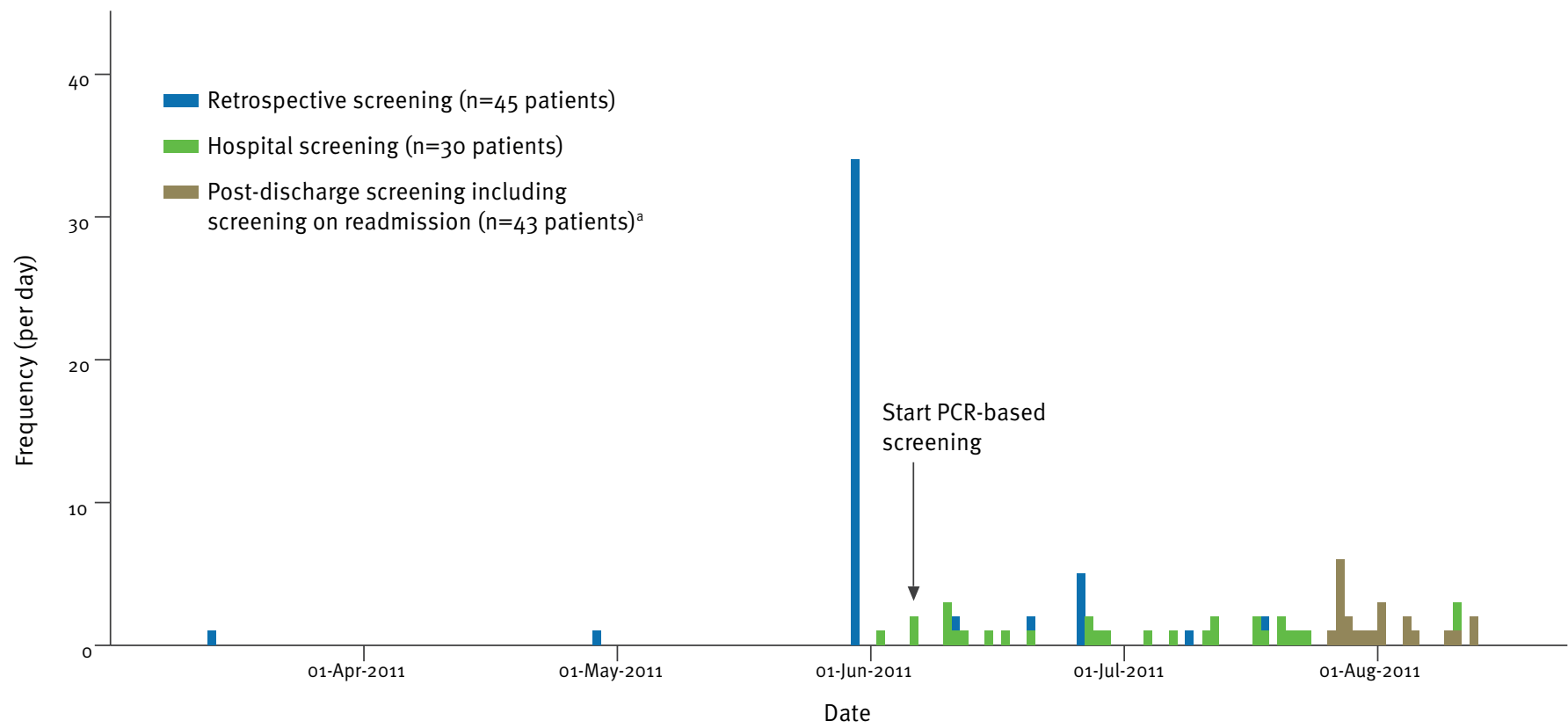

PCR: polymerase chain reaction.

The first two patients were detected in March and April 2011, but notified in May 2011, by other laboratories and are included in the retrospective analysis. Before 10 June PCRs were already performed, but the automated PCR was not fully operational until 10 June so this date was considered the start of PCR-based screening. The retrospective detection was performed on stored Klebsiella pneumoniae samples dating back to 1 January 2009, with the oldest positive sample for OXA-48 dating back from 10 September 2010.

a 21 patients detected after 15 August 2011 are not shown. 
Microbiology and antibiotic susceptibility In 55 of 118 patients (47\%) OXA-48 was detected in more than one Enterobacteriaceae species; patients had five $(n=1)$, four $(n=3)$, three $(n=13)$, or two $(n=38)$ different species. OXA-48 was most frequently identified in K. pneumoniae $(n=99$ patients, $83.9 \%)$ and E. coli $(\mathrm{n}=58$ patients, $49.2 \%$ ) (Table 3 ).

\section{TABLE 3}

Characteristics of OXA-48 positive patients during an outbreak of OXA-48 positive Enterobacteriaceae in a hospital, Rotterdam, the Netherlands, 2009-2011 ( $\mathrm{n}=118$ )

\begin{tabular}{|c|c|}
\hline $\begin{array}{l}\text { Characteristics of OXA- } 48 \text { positive } \\
\text { patients }\end{array}$ & $\begin{array}{c}\text { Patients with OXA-48 } \\
\text { Enterobacteriaceae } \\
(n=118,494 \\
\text { admissions) }\end{array}$ \\
\hline Sex, n male (\%) & $67(57)$ \\
\hline Age in years, median (IQR) & $70.6(60.7-78.0)$ \\
\hline $\begin{array}{l}\text { Median length of stay in days per } \\
\text { admission (IQR) }\end{array}$ & $4(1-14)$ \\
\hline $\begin{array}{l}\text { Median number of admissions during } \\
\text { outbreak period (IQR) }\end{array}$ & $3(2-6)$ \\
\hline $\begin{array}{l}\text { Number of patients admitted to ICU } \\
\text { during outbreak period (\%) }\end{array}$ & $52(44)$ \\
\hline \multicolumn{2}{|l|}{ Number of admissions per specialty (\%) } \\
\hline Internal medicine & $160(32)$ \\
\hline Surgery & $119(24)$ \\
\hline Gastro-enterology & $43(9)$ \\
\hline Cardiology & $37(7)$ \\
\hline Urology & $36(7)$ \\
\hline Pulmonology & $27(5)$ \\
\hline Other & $72(15)$ \\
\hline \multicolumn{2}{|c|}{ Combination of OXA- 48 positive organisms found, $n$} \\
\hline Only Klebsiella pneumoniae & 51 \\
\hline K. pneumoniae and Escherichia coli & 25 \\
\hline K. pneumoniae and other ${ }^{\mathrm{a}}$ & 6 \\
\hline K. pneumoniae and E. coli and other ${ }^{\mathrm{a}}$ & 17 \\
\hline Only E. coli & 9 \\
\hline E. coli and other ${ }^{\mathrm{a}}$ & 7 \\
\hline Only other & 3 \\
\hline $\begin{array}{l}\text { Infection with OXA- } 48 \text { positive } \\
\text { Enterobacteriaceae, } \mathrm{n}(\%)\end{array}$ & $21(18)$ \\
\hline \multicolumn{2}{|l|}{$\begin{array}{l}\text { Patients infected by infection site, } \\
\mathrm{n} \text { ( } \mathrm{n} \text { with positive blood culture) }\end{array}$} \\
\hline Urinary & $5(3)$ \\
\hline Pulmonary & $9(5)$ \\
\hline Abdominal & $5(5)$ \\
\hline Vascular & $1(1)$ \\
\hline Osteomyelitis & $1(1)$ \\
\hline $\begin{array}{l}30 \text { day mortality after first positive } \\
\text { isolate, } \mathrm{n}(\%)\end{array}$ & $19(16)$ \\
\hline $\begin{array}{l}\text { Infection: } \\
\text { deaths/patients infected (\%) }\end{array}$ & $6 / 21(29)$ \\
\hline $\begin{array}{l}\text { Colonisation: } \\
\text { deaths/patients colonised (\%) }\end{array}$ & 13/97 (13) \\
\hline
\end{tabular}

ICU: intensive care unit; IQR: interquartile range.

a Other organisms included Klebsiella oxytoca $(n=8)$, Enterobacter cloacae $(n=7)$, Morganella morganii $(n=6)$, Citrobacter freundii $(n=3)$, Serratia marcescens $(n=3)$, Enterobacter aerogenes $(n=2)$, Citrobacter braakii, Citrobacter farmeri, Citrobacter koseri, Citrobacter youngae, Escherichia fergusonii, Klebsiella ozaenae, Kluyvera species and Raoultella planticola (all $\mathrm{n}=1$ ).
Depending on breakpoints used, at least $85 \%$ of OXA48 isolates ( $K$. pneumoniae and $E$. coli) were susceptible to meropenem and amikacin (Table 4 ).

All OXA-48 K. pneumoniae isolates with CTX-M group 1 ESBL had CTX-M-15 $(n=64)$. Of these 64 patients, 18

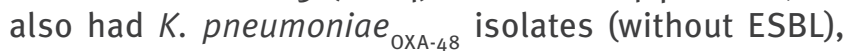
and both isolates were used to describe antibiotic susceptibility (Table 4).

In K. pneumoniae the combination of OXA-48 and CTX$M-15$ was associated with resistance to cefepime, cefotaxime, and gentamicin. Using various Clinical and Laboratory Standards Institute (CLSI) and European Committee on Antimicrobial Susceptibility Testing (EUCAST) breakpoints, susceptibility to imipenem of K. pneumoniaeOXA-48 isolates (without ESBL) ranged from $12 \%$ (CLSI 2010 updated breakpoints [23]) to $83 \%$ (CLSI 2006 breakpoints [24]) and ranged from 3\% to $72 \%$, respectively, for $K$. pneumoniae $\mathrm{O}_{\mathrm{XA}-48 ; \mathrm{CTX}-\mathrm{M}-15}$. All K. pneumoniae OXA- 48 isolates tested $(n=108)$ were nonsusceptible to ertapenem (MIC >0.25 mg/L), as were 45 of 49 E. coli (Table 4).

\section{Molecular epidemiology of Klebsiella pneumoniae}

$285 K$. pneumoniae isolates from patients admitted during the outbreak were included for analysis; 62 OXA-48/CTX-M-1 group, 158 CTX-M-1 group (without OXA-48), 49 with OXA-48 (without CTX-M-1 group ESBL) and 16 with non-CTX-M-1 group ESBL. Of these, 203 isolates were considered to be clonally linked; 62 OXA-48/CTX-M-15, 107 CTX-M-1 group (without OXA48) and 34 with OXA-48 (without CTX-M-1 group ESBL) (Figure 3 and 4).

Among the 82 non-outbreak type $K$. pneumoniae isolates, 50 different types were detected, yielding one cluster of seven isolates (OXA-48 negative, CTX-M-1 group positive $(n=5)$, OXA-48 negative, non-CTX-M-1 group ESBL $(n=2)$ ), one of five (all OXA-48 negative, CTX-M-1 group positive), six of three ( 4 clusters OXA-48 negative, CTX-M-1 group positive; 1 cluster with OXA48 negative non-CTX-M-1 group or CTX-M-1 group ESBL positive; and 1 cluster with 2 OXA-48 positive ESBL negative and 1 OXA-48 negative non-CTX-M-1 group ESBL positive), 10 of two isolates and 32 unique isolates. There were no clusters with more than two OXA48 positive isolates among the non-outbreak isolates.

From September 2010 onwards clonal types $K$. pneumoniae $_{\text {OXA-48;CTX-M-15 }}$ were identified, followed by an increase in clonal types with OXA-48 but without CTX-M-15 in June 2011. These findings are compatible with cross-transmission of clonal $K$. pneumoniae with considerable heterogeneity in the presence of OXA48 and CTX-M-15. OXA-48 encoding plasmids from 39 of 41 isolates (95\%) belonging to 13 different species obtained during the outbreak from 15 patients were identical (based on pMLST), suggesting inter-species transmission of plasmids. 


\section{TABLE 4}

Susceptibility of first OXA-48 positive ESBL positive and negative isolate per patient per organism as determined by Vitek 2 during an outbreak of OXA-48 positive Enterobacteriaceae, Rotterdam, the Netherlands, 2009-2011 (n=118 patients,

171 isolates)

\begin{tabular}{|c|c|c|c|c|c|c|c|c|c|}
\hline \multirow[t]{2}{*}{ Antibiotic } & \multirow[t]{2}{*}{$\begin{array}{l}\text { Breakpoint }^{\mathrm{a}} \\
(\mathrm{mg} / \mathrm{L})\end{array}$} & \multicolumn{2}{|c|}{$\begin{array}{l}\text { K. pneumoniae } \\
\text { OXA-48, } \\
\text { no ESBL }(n=52)\end{array}$} & \multicolumn{2}{|c|}{$\begin{array}{c}\text { K. pneumoniae OXA- } \\
48 \text { and CTX-M-15 } \\
(\mathrm{n}=64)\end{array}$} & \multicolumn{2}{|c|}{$\begin{array}{c}\text { E. coli OXA-48, } \\
\text { no ESBL } \\
(\mathrm{n}=49)\end{array}$} & \multicolumn{2}{|c|}{$\begin{array}{l}\text { E. coli OXA-48 } \\
\text { and CTX-M-15 } \\
\qquad(n=6)\end{array}$} \\
\hline & & $\mathrm{MIC}_{90}$ & $\mathrm{~S}, \mathrm{n}(\%)$ & $\mathrm{MIC}_{90}$ & $\mathrm{~S}, \mathrm{n}(\%)$ & $\mathrm{MIC}_{90}$ & S, n (\%) & $\mathrm{MIC}_{90}$ & S, n (\%) \\
\hline Meropenem & & 2 & & $\geq 16$ & & 1 & & 1 & \\
\hline Breakpoint CLSI 2010-U & 1 & - & $44(85)$ & - & $47(73)$ & - & $49(100)$ & - & $6(100)$ \\
\hline Breakpoint EUCAST & 2 & - & 49 (94) & - & $49(77)$ & - & $49(100)$ & - & $6(100)$ \\
\hline Breakpoint CLSI 2006 & 4 & - & 49 (94) & - & $49(77)$ & - & $49(100)$ & - & $6(100)$ \\
\hline Imipenem & & 8 & & $\geq 16$ & & 2 & & 4 & \\
\hline Breakpoint CLSI 2010-U & 1 & - & $6(12)$ & - & $2(3)$ & - & $35(71)$ & - & $1(17)$ \\
\hline Breakpoint EUCAST & 2 & - & $18(35)$ & - & $15(23)$ & - & $46(94)$ & - & $3(50)$ \\
\hline Breakpoint CLSI 2006 & 4 & - & $43(83)$ & - & $46(72)$ & - & $47(96)$ & - & $6(100)$ \\
\hline Ertapenem & $0.25^{\mathrm{b}}$ & 32 & $\mathrm{o}(\mathrm{o})^{\mathrm{c}}$ & $\geq 32$ & $o(0)^{d}$ & 2 & $4(8)^{e}$ & 2 & $o(0)^{f}$ \\
\hline Amikacin & 8 & $\leq 2$ & $51(98)$ & 8 & $61(95)$ & $\leq 2$ & $47(96)$ & 16 & $5(83)$ \\
\hline Ciprofloxacin & 0.5 & $\geq 4$ & $16(31)$ & $\geq 4$ & $\mathrm{o}(\mathrm{o})$ & $\geq 4$ & $42(86)$ & $\geq 4$ & $1(17)$ \\
\hline $\begin{array}{l}\text { Trimethoprim/ } \\
\text { sulfamethoxazole }\end{array}$ & 2 & $\geq 16$ & $14(27)$ & $\geq 16$ & o (o) & $\geq 16$ & $43(88)$ & $\geq 16$ & $1(17)$ \\
\hline Gentamicin & 2 & $\geq 16$ & $45(87)$ & $\geq 16$ & $\mathrm{o}(0)$ & 8 & $44(90)$ & $\geq 16$ & $4(67)$ \\
\hline Cefotaxime $=$ ceftriaxon & 1 & $\leq 1$ & $47(90)$ & $\geq 64$ & o (o) & 2 & 44 (9o) & $\geq 64$ & o (o) \\
\hline Cefepime & 1 & $\leq 1$ & $52(100)$ & $\geq 64$ & $\mathrm{o}(\mathrm{o})$ & $\leq 1$ & 49 (100) & 32 & $3(50)$ \\
\hline
\end{tabular}

CLSI: Clinical and Laboratory Standards Institute; E. coli: Escherichia coli; ESBL: extended-spectrum beta-lactamases; EUCAST: European committee on antimicrobial susceptibility testing; K. pneumoniae: Klebsiella pneumoniae; MIC: minimum inhibitory concentration; S: susceptible.

a Susceptibility according to EUCAST-breakpoints unless otherwise specified.

b Screening breakpoint.

c Only 49 of the total 52 K. pneumoniae OXA-48, no ESBL were tested for ertapenem.

d Only 59 of the total 64 K. pneumoniae OXA-48 and CTX-M-15 isolates were tested for ertapenem.

e Only 44 of the total 49 E. coli OXA-48, no ESBL isolates were tested for ertapenem.

f Only five of the total six E. coli OXA-48 and CTX-M-15 isolates were tested for ertapenem.

\section{FIGURE 4}

Epidemic curve of outbreak strain Klebsiella pneumoniae isolates producing extended-spectrum beta-lactamases (ESBL) and/or OXA-48, Rotterdam, the Netherlands, 2009-2012 ( $\mathrm{n}=203$ isolates)

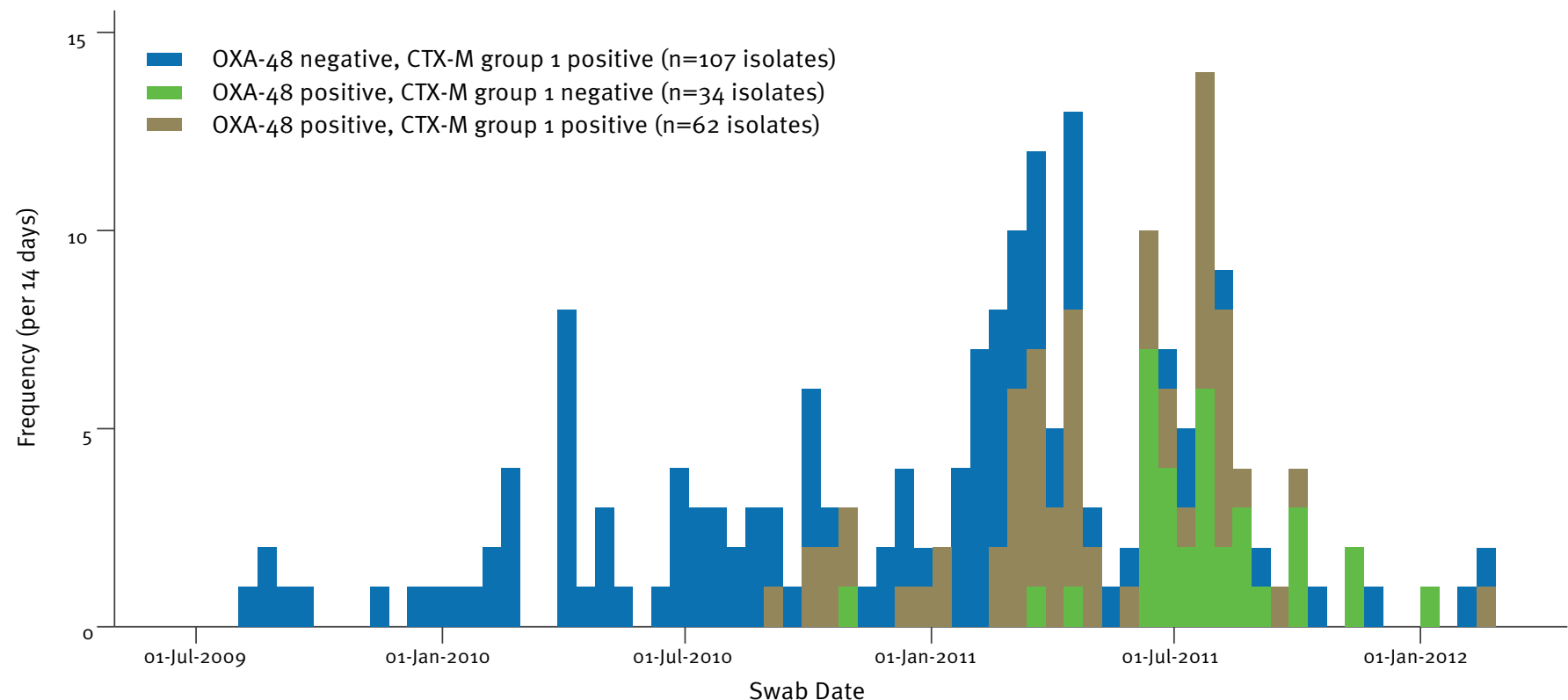

Isolates included in the Figure, detected after 18 July 2011, are from patients admitted during the outbreak period, but screened after the outbreak period in post-discharge and re-admission screening. 


\section{FIGURE 3}

AFLP-typing of 285 Klebsiella pneumoniae isolates, during an outbreak of OXA-48 positive Enterobacteriaceae, Rotterdam, the Netherlands, 2009-2012

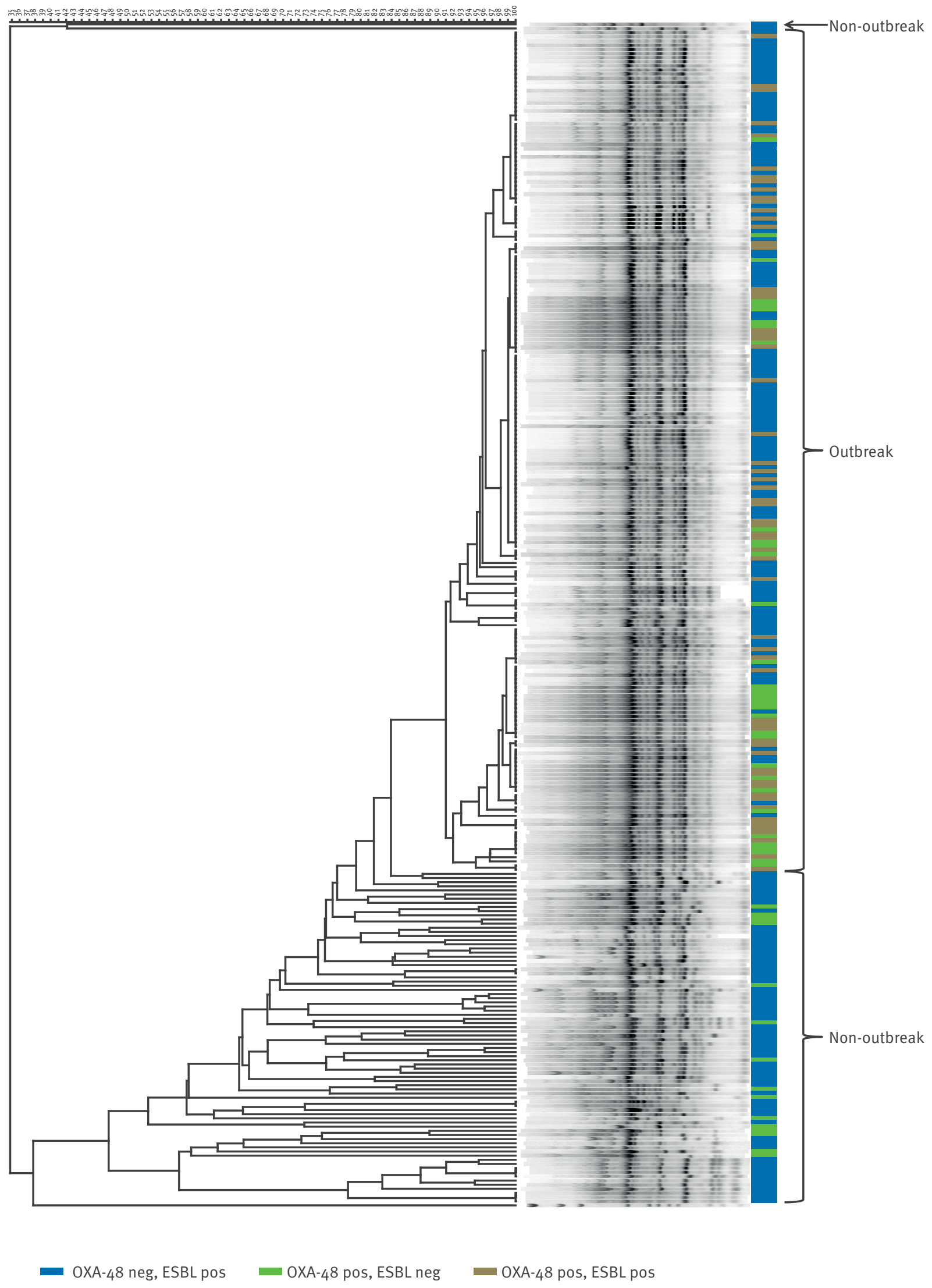

AFLP: amplified fragment length polymorphism; ESBL: extended-spectrum beta-lactamases; neg: negative; pos: positive. Outbreak isolates are based on $\geq 85 \%$ similarity of AFLP patterns (Dice). 
One $K$. pneumoniae outbreak isolate was sequenced and categorised as ST395, and 147 isolates also underwent pulsed-field gel electrophoresis (PFGE) typing, yielding comparable results to AFLP typing (with regard to classification as outbreak-related or not) for 143 isolates (data not shown).

\section{Discussion}

We describe here the successful control of a large hospital outbreak of OXA-48 producing Enterobacteriaceae, which started with nosocomial transmission of $K$. pneumoniae $_{\text {CTX-M-15 }}$ that apparently acquired OXA-48. Among outbreak strains, there was considerable inter- and intra-species heterogeneity in antibiotic susceptibility and resistance genes, and circumstantial evidence of cross-species transfer of OXA-48 containing plasmids. Before the hospital-wide implementation of classical infection control measures and large-scaled PCR-based screening for carriage with OXA-48 containing bacteria, as described here, the outbreak had persisted, partly unnoticed and insufficiently controlled, for two years.

In absence of other resistance genes, OXA-48 expression usually results in low-level resistance, which may hamper laboratory detection. Co-production of ESBL and changes in permeability and in efflux pumps are usually needed for higher resistance levels [2]. In this hospital CLSI 2006 breakpoints were used during the outbreak period, which implied that MICs for imipenem and meropenem $\leq 4 \mathrm{mg} / \mathrm{L}$ were considered susceptible. Although the susceptibility breakpoint for both antibiotics had been reduced to MIC $\leq 1 \mathrm{mg} / \mathrm{L}$ by CLSI in 2010 , even at these new breakpoints $78 \%(91 / 116)$ and $7 \%(8 / 116)$ of $K$. pneumoniae ${ }_{\mathrm{OXA}-48}$, and $100 \%(55 / 55)$ and $65 \%(36 / 55)$ of $E$. coli ${ }_{0 \times A-48}$ isolates would have been considered susceptible to meropenem and imipenem, respectively. Based on EUCAST guidelines $84 \%$ $(98 / 116)$ and $28 \%(33 / 116)$ of $K$. pneumoniae OXA-48 $_{\text {, }}$ and $100 \%(55 / 55)$ and $89 \%(49 / 55)$ of $E$. coli $i_{\mathrm{OXA}-48}$ isolates would have been considered susceptible to meropenem and imipenem, respectively. Ertapenem susceptibility testing (using the screening breakpoint of $>0.25 \mathrm{mg} / \mathrm{L}$ ) would have detected 128 of the 132 OXA-48 isolates ( $97 \%$ ) with meropenem MIC $\leq 1 \mathrm{mg} / \mathrm{L}$, and is, therefore, the carbapenem of choice when screening for OXA-48. This phenotypic variability among OXA-48 containing bacteria represents a major threat for unnoticed spread of this resistance gene. Large-scale gene detection, routine testing for ertapenem susceptibility, or novel phenotypic methods (such as chromogenic media) may be needed to prevent unnoticed dissemination. The latter methods however, yield considerable variation in sensitivity for OXA-48 detection, with $84 \%$ for carbapenem-resistant Enterobacteriaceae (CRE) agar [25] and $100 \%$ for the Carbapenemase Nordmann-Poirel (Carba NP) test [26].

As with most hospital outbreaks, the simultaneous implementation of interventions and lack of quantitative evaluation of adherence to these interventions precludes a truly scientific analysis of their effectiveness. Our findings suggest that contact isolation measures using single rooms with individual sanitary facilities attributed to controlling transmission. This was feasible as two old hospital buildings had transmigrated to a new building with more single rooms, shortly before outbreak detection. Naturally this migration might have influenced infection control also in other ways, such through differences in building structure that could not be quantified. Furthermore, widespread knowledge of an ongoing outbreak (with significant attention by the lay press) since June 2011 may also have contributed to better adherence to basic infection control measures. Based on the data available there was no evidence of environmental contamination or carriage among healthcare personnel with the outbreak strain.

The largest proportion of patients had been treated in ICU, suggesting that this was the epicentre of the outbreak. Yet, detection bias due to higher culture frequency is likely. Moreover, most identification was based on either retrospective or post-discharge screening and many patients had been treated in different wards. Therefore, more sophisticated analyses are needed to determine the relative importance of different wards in the outbreak.

Selective decontamination of the digestive tract (SDD) has been associated with eradication (or suppression) of resistant Gram-negative bacteria in the gut [27-29]. SDD (using tobramycin and polymyxin E) was introduced in the ICU in hospital A in May 2011, just before the transition to the new facility and hospitalwide implementation of control measures. SDD was not intended to eliminate OXA-48 carriage, but was implemented for all eligible patients because of its presumed benefits on patient outcome [30]. Because of the timing of events it will be difficult to quantify the role of SDD in controlling this outbreak. Preliminary analyses do not identify an immediate change in the numbers of hospitalised OXA-48 carriers (data not shown).

The first detected OXA-48 isolate originated from September 2010, but it cannot be excluded that OXA48 was already present in the hospital before that date. Although many isolates with an ESBL phenotype had been stored, isolates with minimally elevated MICs for carbapenems were not. Furthermore, screening for ESBL-carriage was implemented in ICU in June 2010 and in other departments in August 2010, and hospital-wide PCR-based screening for OXA-48 carriage started in June 2011. In addition, OXA-48 carriers may lose their carrier status which will reduce detection in post-discharge screening. Therefore, underreporting of OXA-48 carriage is likely. Yet, we do not assume OXA-48 was circulating for many years in the hospital. The oldest OXA-48 isolate originated from a culture obtained in September 2010, and more than 40 isolates clonally linked to the outbreak strain (Figure 3) were detected before, but none of them harboured OXA-48. 
This strongly suggests widespread circulation of the ESBL-producing strain without OXA-48 in the hospital before September 2010. Indeed, our hypothesis is that the ESBL-producing outbreak strain was circulating in the hospital and acquired the OXA-48-containing plasmid from another patient, most probably coming from an endemic region. Risk factors for OXA-48 acquisition, duration of carriage of OXA-48 after hospital discharge and its transmissibility in the community setting are currently investigated.

In the early phases of outbreak containment several important decisions had to be made, in the absence of scientific evidence. The tentative start of nosocomial OXA-48 transmission needed to be defined on incomplete data. Therefore, a 14-month margin after the 'oldest' isolate, which was identified at the end of May 2011, was taken. Furthermore, it was known that Shewanella spp. can carry OXA-48. Yet, the outbreak was considered to be caused by $K$. pneumoniae only. As we detected patients with non-Klebsiella Enterobacteriaceae carrying OXA-48 after implementing PCR-based screening of rectal swabs, it was subsequently decided to include Enterobacteriaceae (but not Shewanella spp.) in our case definition. Risk categorisation was based on proximity of patients to identified OXA-48 carriers. For feasibility reasons we decided to base this risk on 'room sharing' only, although there are many more potential risk factors. Finally, based on limited data it was decided not to further investigate a potential role of colonised healthcare workers in this outbreak, nor to change environmental cleaning procedures and antibiotic policies. Enforced cleaning and antimicrobial stewardship were considered instrumental in controlling a large OXA-48 outbreak in Spain [31]. In our setting, there were no documented events of nosocomial transmission of OXA-48 Enterobacteriaceae after 18 July 2011 without these measures. We have not performed a detailed analysis of antibiotic use, but in the cohort of patients who were admitted during the outbreak period and who were tested for OXA48 carriage (and considered non-carrier based on test results; $n=11,386$ ) carbapenem use was 0.67 days per 100 admission days. Unfortunately, days in ICU could not be included in this analysis.

After outbreak detection other healthcare facilities in the region were immediately informed and advised to screen patients who had been admitted to hospital A during the outbreak period. Some hospitals also isolated such patients awaiting culture results. To the best of our knowledge, there were no additional carriers identified through screening in other hospitals. There was one secondary case of OXA-48 carriage in a long-term care facility that had received an OXA-48 carrier after treatment in hospital A. Extensive screening failed to identify further spread.

Some important lessons can be learned from this outbreak. First, uncontrolled spread of ESBL-producing bacteria, even at relatively low levels, can turn into an outbreak of carbapenemase-producing bacteria, most probably after horizontal gene transfer. The likelihood of horizontal gene transfer will increase if admission rates of patients with unidentified carriage with such bacteria increases. Currently, this rate appears to be low in the Netherlands and Belgium. In this hospital four new OXA-48 carriers, unrelated to the outbreak, were discovered due to extensive screening of 1,921 patients. In Belgium, eight OXA-48 positive isolates were detected among 4,564 Enterobacteriaceae isolates (one isolate per patient) [32]. Second, because of the large heterogeneity of antibiotic susceptibilities routine phenotypic detection tests may be insufficient for identification of all isolates involved in an outbreak. Third, once detected, this large outbreak could be controlled - in short time - presumably with classical infection control measures, although it was impossible to quantify the contribution of individual measures and the role of special circumstances, such as the relocation of the hospital into a new facility. Because the outbreak might have remained unnoticed for some time however, the resources needed to identify its extent, as well as to screen potential carriers of OXA-48 Enterobacteriaceae were extensive $(74,884$ PCRs were performed for OXA-48 screening in 2011 and 2012). We, therefore, recommend implementing risk-stratified screening for carriage of these highly resistant bacteria, followed by barrier precautions for carriers detected, in order to prevent the need of costly control measures after detection of an unnoticed outbreak. Risk factor analyses for OXA-48 carriage are, therefore, warranted.

\section{Acknowledgements}

We had no external funding sources. Hospital A received assistance in outbreak control from M.E.A. de Kraker, S.C. de Greeff, H.A. Bijlmer and H. Grundmann from the National Institute of Health and the Environment, and A. Troelstra, C.A.M. de Graaf-Miltenburg and M.J.M. Bonten from the department of Medical Microbiology of the University Medical Center Utrecht.

\section{Conflict of interest}

None declared.

\section{Authors' contributions}

The investigators were responsible for data collection, data analysis, data interpretation, and writing of the report. The corresponding author had full access to all the data in the study and had final responsibility for the decision to submit for publication.

\section{References}

1. Gupta N, Limbago BM, Patel JB, Kallen AJ. Carbapenemresistant Enterobacteriaceae: epidemiology and prevention. Clin Infect Dis. 2011;53(1):60-7. http://dx.doi.org/10.1093/cid/ cir202

2. Nordmann P, Naas T, Poirel L. Global spread of Carbapenemase-producing Enterobacteriaceae. Emerg 
Infect Dis. 2011;17(10):1791-8. http://dx.doi.org/10.3201/ eid1710.110655

3. Pitout JD, Laupland KB. Extended-spectrum beta-lactamase producing Enterobacteriaceae: an emerging public-health concern. Lancet Infect Dis. 2008;8(3):159-66. http://dx.doi. org/10.1016/S1473-3099(08)70041-0

4. Poirel L, Héritier C, Tolün V, Nordmann P. Emergence of oxacillinase-mediated resistance to imipenem in Klebsiella pneumoniae. Antimicrob Agents Chemother. 2004;48(1):15-22. http://dx.doi.org/10.1128/AAC.48.1.15-22.2004

5. Nagano N, Endoh Y, Nagano Y, Toyama M, Matsui M, Shibayama K, et al. First report of OXA-48 carbapenemaseproducing Klebsiella pneumoniae and Escherichia coli in Japan from a patient returned from Southeast Asia. Jpn J Infect Dis. 2013;66(1):79-81. http://dx.doi.org/10.7883/yoken.66.79

6. Ktari S, Mnif B, Louati F, Rekik S, Mezghani S, Mahjoubi F et al. Spread of Klebsiella pneumoniae isolates producing OXA-48 beta-lactamase in a Tunisian university hospital. J Antimicrob Chemother. 2011;66(7):1644-6. http://dx.doi.org/10.1093/jac/ dkr181

7. Moquet O, Bouchiat C, Kinana A, Seck A, Arouna O, Bercion R et al. Class D OXA-48 carbapenemase in multidrug-resistant enterobacteria, Senegal. Emerg Infect Dis. 2011;17(1):143-4. http://dx.doi.org/10.3201/eid1701.100244

8. Poirel L, Ros A, Carrer A, Fortineau N, Carricajo A, Berthelot $P$ et al. Cross-border transmission of OXA-48-producing Enterobacter cloacae from Morocco to France. J Antimicrob Chemother. 2011;66(5):1181-2. http://dx.doi.org/10.1093/jac/ dkro23

9. Brink AJ, Coetzee J, Corcoran C, Clay CG, Hari-Makkan D, Jacobson RK, et al. Emergence of OXA-48 and OXA-181 carbapenemases among Enterobacteriaceae in South Africa and evidence of in vivo selection of colistin resistance as a consequence of selective decontamination of the gastrointestinal tract. J Clin Microbiol. 2013;51(1):369-72. http://dx.doi.org/10.1128/JCM.02234-12

10. Cuzon G, Ouanich J, Gondret R, Naas T, Nordmann P. Outbreak of OXA-48-positive carbapenem-resistant Klebsiella pneumoniae isolates in France. Antimicrob Agents Chemother 2011;55(5):2420-3. http://dx.doi.org/10.1128/AAC.01452-10

11. Glupczynski Y, Huang TD, Bouchahrouf W, Rezende de Castro R, Bauraing C, Gérard M et al. Rapid emergence and spread of OXA-48-producing carbapenem-resistant Enterobacteriaceae isolates in Belgian hospitals. Int J Antimicrob Agents. 2012;39(2):168-72. http://dx.doi.org/10.1016/j. ijantimicag.2011.10.005

12. Pitart C, Solé M, Roca I, Fàbrega A, Vila J, Marco F. First outbreak of a plasmid-mediated carbapenem-hydrolyzing OXA-48 beta-lactamase in Klebsiella pneumoniae in Spain. Antimicrob Agents Chemother. 2011;55(9):4398-401. http:// dx.doi.org/10.1128/AAC.00329-11

13. Lascols C, Peirano G, Hackel M, Laupland KB, Pitout JD. Surveillance and molecular epidemiology of Klebsiella pneumoniae isolates that produce carbapenemases: first report of OXA-48-like enzymes in North America. Antimicrob Agents Chemother. 2013;57(1):130-6. http://dx.doi org/10.1128/AAC.01686-12

14. Carrër A, Poirel L, Eraksoy H, Cagatay AA, Badur S, Nordmann P. Spread of OXA-48-positive carbapenem-resistant Klebsiella pneumoniae isolates in Istanbul, Turkey. Antimicrob Agents Chemother. 2008;52(8):2950-4. http://dx.doi.org/10.1128/ AAC.01672-07

15. Carrër A, Poirel L, Yilmaz M, Akan OA, Feriha C, Cuzon G et al. Spread of OXA-48-encoding plasmid in Turkey and beyond. Antimicrob Agents Chemother. 2010;54(3):1369-73. http:// dx.doi.org/10.1128/AAC.01312-09

16. Crémet L, Bourigault C, Lepelletier D, Guillouzouic A, Juvin $M E$, Reynaud A et al. Nosocomial outbreak of carbapenemresistant Enterobacter cloacae highlighting the interspecies transferability of the blaOXA-48 gene in the gut flora. J Antimicrob Chemother. 2012;67(4):1041-3. http://dx.doi. org/10.1093/jac/dkr547

17. Levast M, Poirel L, Carrër A, Deiber M, Decroisette E, Mallaval FO et al. Transfer of OXA-48-positive carbapenem-resistant Klebsiella pneumoniae from Turkey to France. J Antimicrob Chemother. 2011;66(4):944-5. http://dx.doi.org/10.1093/jac/ dkq504

18. Dutch Working party on Infection Prevention. Measures to prevent the transmission of highly resistant microorganisms (HRMO). 2005. [Accessed 17 Aug 2012]. Available from: http:// www.wip.nl/UK/free_content/Richtlijnen/HRMO.pdf

19. Poirel L, Walsh TR, Cuvillier V, Nordmann P. Multiplex PCR for detection of acquired carbapenemase genes. Diagn Microbiol Infect Dis. 2011;70(1):119-23. http://dx.doi.org/10.1016/j. diagmicrobio.2010.12.002
20. Nerderlandse Vereniging voor Medische Microbiologie (NVMM). Guideline Laboratory detection of highly resistant microorganisms, version 1.0. 2011. Leeuwarden:NVMM; 2011. [Accessed 5 Mar 2014]. Available from: http://www.med-info. $\mathrm{nl} /$ Richtlijnen/Infectieziekten/Laboratory\%2odetection $\% 20$ of $\% 2$ highly \%2oresistant $\% 20$ microorganisms\%20(HRMO).pdf

21. Van der Zee A, Steer N, Thijssen E, Nelson J, van't Veen A, Buiting A. Use of multienzyme multiplex PCR amplified fragment length polymorphism typing in analysis of outbreaks of multiresistant Klebsiella pneumoniae in an intensive care unit. J Clin Microbiol. 2003;41(2):798-802. http://dx.doi. org/10.1128/JCM.41.2.798-802.2003

22. Donnarumma F, Indorato C, Mastromei G, Goti E, Nicoletti $P$, Pecile P, et al. Molecular analysis of population structure and antibiotic resistance of Klebsiella isolates from a threeyear surveillance program in Florence hospitals, Italy. Eur J Clin Microbiol Infect Dis. 2012;31(3):371-8. http://dx.doi. org/10.1007/s10096-011-1319-6

23. Clinical and Laboratory Standards Institute (CSLI). Performance Standards for Antimicrobial Susceptibility Testing; Twentieth Informational Supplement (June 2010 Update). Wayne, PA: CSLI; 2010. CLSI document M100-S20-U.

24. Clinical and Laboratory Standards Institute (CSLI). Performance Standards for Antimicrobial Susceptibility Testing; Sixteenth Informational Supplement. Wayne, PA: CSLI; 2006. CLSI document M100-S16.

25. Cohen Stuart J, Voets G, Rottier W, Voskuil S, Scharringa J, Van Dijk K, et al. Evaluation of the Oxoid Brilliance ${ }^{\mathrm{TM}}$ CRE Agar for the detection of carbapenemase-producing Enterobacteriaceae. Eur J Clin Microbiol Infect Dis. 2013;32(11):1445-9. http:// dx.doi.org/10.1007/s10096-013-1896-7

26. Nordmann P, Poirel L, Dortet L. Rapid detection of carbapenemase-producing Enterobacteriaceae. Emerg Infect Dis. 2012;18(9):1503-7. http://dx.doi.org/10.3201/ eid1809.120355

27. Saidel-Odes L, Polachek H, Peled N, Riesenberg K, Schlaeffer $\mathrm{F}$, Trabelsi $\mathrm{Y}$ et al. A randomized, double-blind, placebocontrolled trial of selective digestive decontamination using oral gentamicin and oral polymyxin $\mathrm{E}$ for eradication of carbapenem-resistant Klebsiella pneumoniae carriage. Infect Control Hosp Epidemiol. 2012;33(1):14-9. http://dx.doi. org/10.1086/663206

28. Brun-Buisson C, Legrand P, Rauss A, Richard C, Montravers $\mathrm{F}$, Besbes $\mathrm{M}$ et al. Intestinal decontamination for control of nosocomial multiresistant gram-negative bacilli. Study of an outbreak in an intensive care unit. Ann Intern Med. 1989;110(11):873-81. http://dx.doi. org/10.7326/0003-4819-110-11-873

29. Oostdijk EA, de Smet AM, Kesecioglu J, Bonten MJ. Decontamination of cephalosporin-resistant Enterobacteriaceae during selective digestive tract decontamination in intensive care units. J Antimicrob Chemother. 2012;67(9):2250-3. http://dx.doi.org/10.1093/jac/ dks187

30. de Smet AM, Kluytmans JA, Cooper BS, Mascini EM, Benus RF, van der Werf TS, et al. Decontamination of the digestive tract and oropharynx in ICU patients. N Engl J Med. 2009;360(1):2031. http://dx.doi.org/10.1056/NEJMoa0800394

31. Pa-o-Pardo IR, Ruiz-Carrascoso G, Navarro-San Francisco C, Gómez-Gil R, Mora-Rillo M, Romero-Gómez MP, et al. Infections caused by OXA-48-producing Klebsiella pneumoniae in a tertiary hospital in Spain in the setting of a prolonged, hospital-wide outbreak. J Antimicrob Chemother. 2013;68(1):89-96. http://dx.doi.org/10.1093/jac/dks364

32. Huang TD, Berhin C, Bogaerts P, Glupczynski Y; multicentre study group. Prevalence and mechanisms of resistance to carbapenems in Enterobacteriaceae isolates from 24 hospitals in Belgium. J Antimicrob Chemother. 2013;68(8):1832-7. http:// dx.doi.org/10.1093/jac/dkto96 